\title{
Synthesis of a Multi-beam Dual Reflectarray Antenna Using Genetic Algorithms
}

\author{
Ciaran Geaney , Jianwei Sun, Sean Victor Hum
}

\begin{abstract}
This paper presents the results of an initial study on the use of genetic algorithms to synthesize multi-beam patterns for dual-reflectarray antennas operating with a single feed per beam architecture. Under the simple two-dimensional center-fed case considered, the method shows promise when compared to a traditional bifocal synthesis method with regard to spillover efficiency.
\end{abstract}

\section{INTRODUCTION}

To increase capacity, modern Ka-band communications satellites produce multiple high gain overlapping spot beams that alternate in frequency and polarization [1]. A simple architecture to produce such coverage is single feed per beam (SFB), in which each beam is produced by a separate feed. However, due to aperture efficiency requirements which impose constraints on feed horn sizes, this scheme generally requires three or four reflectors to produce the correct beam spacing [2]. To reduce the number of apertures required to two, a multiple feed per beam architecture can be used, but at the expense of a complicated beamforming network (eg. [1]). To maintain the simplicity of SFB while using a single main aperture, genetic algorithms (GAs) are studied in this work to produce the required beams using realistically sized non-overlapping feeds with a dual-reflectarray antenna.

\section{GEOMETRY AND ANALYSIS}

For this initial investigation, a center-fed dual reflectarray configuration is chosen and it is shown in Fig. 1. The geometrical parameters are $D_{m}=1.8 \mathrm{~m}, D_{s}=0.6 \mathrm{~m}, d_{m s}=2.27 \mathrm{~m}$, and $d_{f s}=0.5 \mathrm{~m}$. The periodicity of both reflectarrays is $0.5 \lambda_{0}$ at the design frequency of $20 \mathrm{GHz}$ to avoid grating lobes.

Five feeds with diameters and spacing of $54 \mathrm{~mm}$ are considered in the optimization and analysis. The required beam peak locations for these feeds are shown in Table I. Using the single-focus folded optics system with a traditional reflector, shown in Fig. 1, this beam spacing is not possible for this feed size without overlapping the feeds.

To model the feeds a $\cos ^{q} \theta$ model is used, with $q=28$. The analysis and optimization are carried out in $2 \mathrm{D}$, but there are methods available to extend the technique to $3 \mathrm{D}$. The analysis is carried out using an array factor calculation [3], treating each element as an ideal phase-shifter.

\author{
Eduardo Martinez-de-Rioja, Jose A. Encinar
}

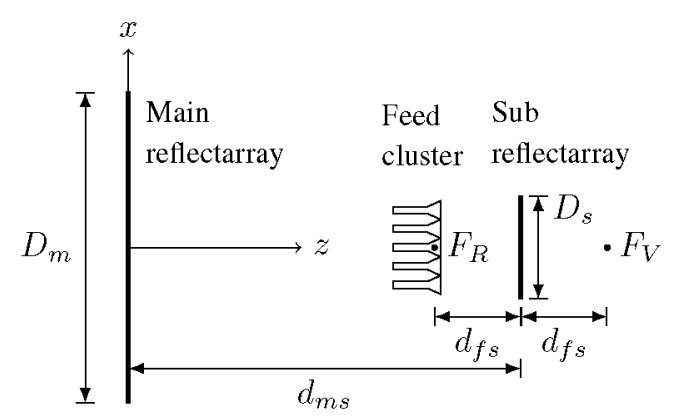

Fig. 1. Symmetric center-fed dual reflectarray geometry with locations of real focus $\left(F_{R}\right)$ and virtual focus $\left(F_{V}\right)$ of reference system shown.

\section{OPTIMIZATION DETAILS}

An issue with applying a traditional bifocal synthesis technique to this problem is that the solutions have poor spillover efficiency [4]. Thus, for the optimization routine spillover was taken into account by calculating the total gain of the system based on an estimate of spillover efficiency. A mask-driven approach was initially pursued whereby individual masks for each beam/feed were specified, and the following cost function that describes the degree of mask violation was used:

$$
\begin{array}{r}
C(\theta)=\sum_{i=1}^{N} \int_{-\pi / 2}^{\pi / 2}\left\{\min \left[M_{U_{i}}(\theta)-G_{i}(\theta), 0\right]^{2}\right. \\
\left.\min \left[G_{i}(\theta)-M_{L_{i}}(\theta), 0\right]^{2}\right\} \mathrm{d} \theta
\end{array}
$$

Here, $i$ indicates the feed/beam number, $N$ is the total number of feeds, $M_{U}(\theta)$ and $M_{L}(\theta)$ are the upper and lower masks, respectively, and $G(\theta)$ is the gain pattern that includes illumination efficiency. Through testing various masks it was found that the best results were obtained by simply imposing a minimum gain at the desired beam direction for each feed.

The GA was initialized with random values for 240 and 80 phases on the main and sub-reflectarray, respectively. However, the optimization was only performed for half of these phases $(120+40)$ due to symmetry. Each phase was represented as a gene encoded by an 8-bit unsigned character. The genes corresponding to the main and sub-reflectarrays were then 

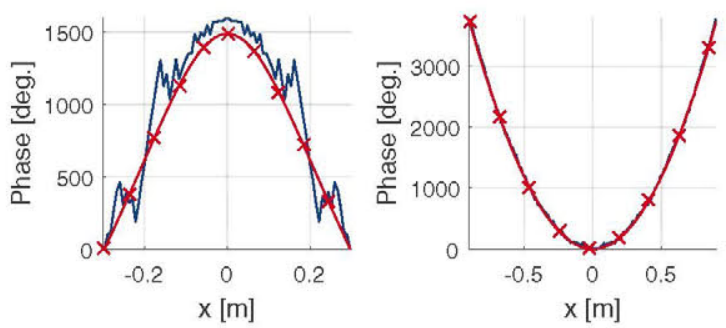

Fig. 2. Required relative phase distributions on sub (left) and main (right) reflectarrays resulting from $\mathrm{GA}(-)$ and bifocal $(\leftarrow)$

concatenated together to produce a chromosome representing a solution to the synthesis problem. A population of 100 chromosomes was found to balance computational resources against comprehensive searching.

At each generation of the GA, the crossover operation was applied with $80 \%$ probability for 50 pairs, which were selected binary-tournament style from the population. The crossover operation was applied separately to the main and sub-reflectarray phases. The mutation operation was then applied after the crossover operations on each chromosome with a $50 \%$ probability. This operation randomly toggled a single bit in a randomly selected gene from the chromosome. Elitism of a single chromosome was also utilized in this GA.

\section{RESULTS AND COMPARISON TO BIFOCAL SYNTHESIS}

The required phase distributions obtained from the GA synthesis are shown in Fig. 2 and compared with a bifocal synthesis [4] based on [5] that was implemented for the same geometry. It is interesting to note that while the methods produce different sub-reflectarray phases, they both produce a main reflectarray with roughly the same $f / D$ ratio. However, the main reflectarray phase from the GA is not purely parabolic and it was found that substituting it with a curve-fit parabola produced beams with worse beam aberration.

In Fig. 3 the $x$-component of the incident field on the main reflectarray is compared for both techniques. Only the fields from one half of the feeds are shown, as the others can be inferred from the symmetry of the geometry considered. It is visible that the amplitude taper produced by the bifocal synthesis is smoother, but the high taper levels on at least one edge of the main reflectarray would produce very high spillover. It was found in earlier investigations that these levels could not be corrected by re-pointing the feeds towards the center of the sub-reflectarray. The GA produces more acceptable edge taper levels that range from $8 \mathrm{~dB}$ to $10 \mathrm{~dB}$ below the peak value.

Fig. 4 shows the resulting normalized radiation patterns from these phase distributions, and pointing errors are shown in Table I. It is visible that the beams are pointed similarly, but the GA beams have slightly higher gains while suffering from increased sidelobe levels. However, it is possible that these results could be improved through using different masks.
TABLE I

DESIRED BEAM LOCATIONS AND POINTING ERROR

\begin{tabular}{|c|c|c|c|}
\hline \multirow[t]{2}{*}{ Feed } & \multirow{2}{*}{$\begin{array}{l}\text { Desired beam } \\
\text { direction [deg.] }\end{array}$} & \multicolumn{2}{|c|}{ Beam pointing error [deg.] } \\
\hline & & GA & Bifocal \\
\hline 2 from center & -1.12 & 0.02 & 0.01 \\
\hline 1 from center & -0.56 & 0.01 & 0.02 \\
\hline Center & 0.00 & 0.01 & 0.01 \\
\hline 1 from center & 0.56 & 0.03 & 0.01 \\
\hline 2 from center & 1.12 & 0.03 & 0.03 \\
\hline
\end{tabular}

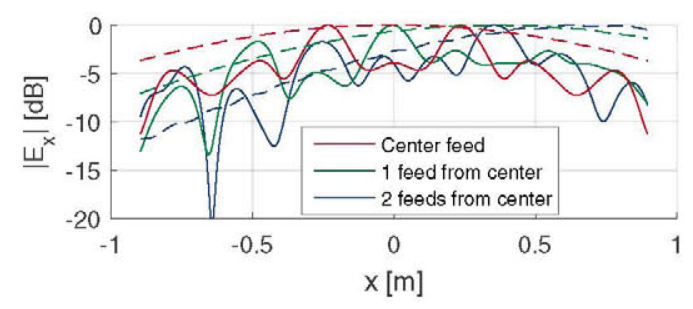

Fig. 3. Main reflectarray illumination. Solid line: GA, dashed line: bifocal

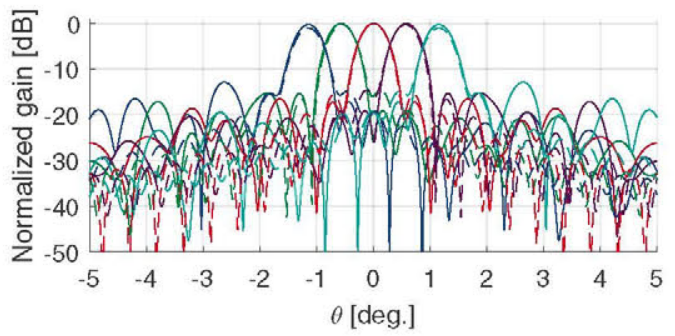

Fig. 4. Normalized radiation patterns from each feed at $20 \mathrm{GHz}$. Solid line: GA, dashed line: bifocal

Although only five feeds and beams have been shown here for clarity, the method is also extendible to cases with more feeds.

\section{CONCLUSION}

An initial study on the use of GAs to synthesize dualreflectarray antennas for multi-beam applications has been performed. In 2D the method shows improvement with regard to spillover efficiency compared to a bifocal synthesis. Further work is under way to improve beam quality, and extend the technique to 3D.

\section{REFERENCES}

[1] M. Schneider, C. Hartwanger, and H. Wolf, "Antennas for multiple spot beam satellites," CEAS Space Journal, vol. 2, no. 1, pp. 59-66, 2011.

[2] E. Amyotte, Y. Demers, V. Dupessey, M. Forest, L. Hildebrand, A. Liang, M. Riel, and S. Sierra-Garcia, "A summary of recent developments in satellite antennas at MDA," in Proc. of the 5th Eur: Conf. on Antennas and Propag. (EUCAP), April 2011, pp. 3203-3207.

[3] C. Tienda, M. Arrebola, J. A. Encinar, and G. Toso, "Analysis of a dualreflect array antenna," IET Microwaves, Antennas Propagation, vol. 5, no. 13, pp. 1636-1645, October 2011.

[4] E. M. de Rioja, J. A. Encinar, C. Geaney, and S. V. Hum, "Study of bifocal dual reflectarray configurations for multi-beam antennas in Kaband," in 2017 IEEE Antennas and Propag. Soc. Int. Symp. (APSURSI), submitted.

[5] C. Rappaport, "An offset bifocal reflector antenna design for wide-angle beam scanning," IEEE Trans. Antennas Propag., vol. 32, no. 11, pp. 1196-1204, Nov 1984. 